\title{
A MULTIPURPOSE AND NOVEL CARRIER FOR DRUG DELIVERY AND TARGETING - VIROSOMES
}

\author{
Kapoor D*, Vyas RB, Lad C, Patel M \\ Dr. Dayaram Patel Pharmacy College, Sardarbaug, Station Road, Bardoli, Dist - Surat, Gujarat, India, Pin-394601 \\ *Corresponding Author'sE-mail id - dev7200@gmail.com, Contact Info - +91-7874223242
}

\begin{abstract}
:
There is presently passionate investigation bustle aimed at the improvement of new delivery systems for vaccines. The aspiration is to identify best possible methods for presenting target antigens to the immune system in a manner that will elicit immune responses appropriate for protection against, or treatment of, a specific disease. Virosomes are biocompatible, biodegradable, nontoxic, and non-autoimmunogenic, attempts have been made to use them as vaccines or adjuvant as well as delivery systems for drugs, nucleic acids, or genes for therapeutic purposes. Influenza virus is the most common virus of choice. There are at present numerous factors that are creating pressure to develop delivery systems for vaccines. First, in the existing regulatory milieu, there is a budding prerequisite to build up vaccines that are very well defined in molecular requisites. Unambiguous targeting and liberation as well as the display of antigens on the surface of Professional antigenpresenting cells (APCs) are key issues in the blueprint and improvement of new-generation vaccines intended at the initiation of both humoral and cell mediated immunity. Prophylactic vaccination in opposition to infectious diseases in general aims at the generation of humoral immune responses to prevent infection. However, 143mmunization with live vaccines bears the peril of causing ailment. For that reason, unconventional vaccine delivery systems are must to produce better immune response. Virosomal technology presents a fresh urbane delivery system to congregate these challenges.On the whole, virosomes guard pharmaceutically active substances from proteolytic dilapidation and low $\mathrm{pH}$ within endosomes, allowing their contents to linger intact when they get in touch with the cytoplasm. This is a foremost benefit of virosomal transporter systems over other drug-delivery vehicles, including liposomal and proteoliposomal carrier systems.
\end{abstract}

Key-Words: Novel Drug Delivery, Virosomes, Vaccine

\section{INTRODUCTION}

A virosome is the reconstituted membrane of an enveloped virus. Many viruses are delimited by a membrane, or shroud, that is resulting from a membrane of the swarm cell; these viruses are produced by budding from a host cell membrane. The envelope thus contains lipids of the host cell and viral proteins. These viral proteins have a purpose in binding to new host cells, and in inducing fusion of a membrane of the new host cell with the viral membrane.

Virosomes are reconstituted viral envelopes that can make available as vaccines and as vehicles for cellular delivery of macromolecules. The panorama of drug delivery and targeting using virosomes is anfascinatingmeadow of research and development. Influenza virus is the most common virus of choice. The triumph of virosomal drug delivery depends on the methods used to prepare the encapsulated bioactive materials and integrate them into the virosomes, as are depiction and formulation of the finished preparation. Virosome technology could potentially be used to deliver peptides, nucleic acids or genes, and drugs like antibiotics, anticancer agents, and steroids. Reconstituted influenza virus envelopes (virosomes) containing the viral hemagglutinin (HA) have attracted consideration as liberation vesicles for cytosolic drug delivery as they acquire membrane fusion bustle. ${ }^{1}$ Virosomes were first primed by Almeida et al., who inserted purified influenza spike proteins into preformed liposomes. ${ }^{2}$ Enveloped creature viruses can send their genome into the cytoplasm of a host cell by fusing with the plasma membrane or with the endosomal membrane. ${ }^{3}$
Virosome technology is a tool for developing novel, predominantly synthetic vaccines against infectious and chronic diseases. A virosome is a virus-like particle that acts as a vaccine carrier and adjuvant (immunity enhancing) system. Vaccines based on virosome technology combine high efficacy with high purity, which means they are effective and safe to use even in infants and individuals with a weakened immune system. A, rabies, tick-borne encephalitis, Japanese B encephalitis and Rift Valley fever ${ }^{4}$. The difficulty in eliciting mucosal immunity with subunit vaccines has directed research in the development of new delivery systems which have improved local and systemic immune responses to mucosal administered antigens $5,6,7,8$

\section{Benefits of Virosomal Drug Delivery}

Virosomes protect pharmaceutically active substances from proteolytic degradation and low $\mathrm{pH}$ within endosomes, allowing their contents to remain intact when they reach the cytoplasm. This is a major advantage of virosomal carrier systems over other drug-delivery vehicles, including liposomal and proteoliposomal carrier systems.Capable drugs are habitually discontinued for the duration ofenhancementfor the reason that they cannot be appropriately delivered to target cells, tissues, and organs. The new cohort of therapeutics alongside cancer or neurodegenerative disorders requires delivery systems that target drugs to precise cell types and host tissues by receptor-mediated uptake and controlled release. Virosomal technology presents a novel sophisticated delivery system to meet these challenges. 
Virosomal technology is accepted by the FDA for bring into play in humans, and has a high security silhouette. Virosomes are biodegradable, biocompatible, and nontoxic, no disease-transmission risk, no autoimmunogenity or anaphylaxis.Virosomes represent an innovative, broadly applicable carrier systemwith various prospective applications for the treatment and prevention ofcancer, neurodegenerative disorders and infectious diseases. Various pharmaceutically active substances like antibiotics, cytostatics, nucleic acids, fungicidesand antigens can be encapsulated into the virosomal carrier. Even thesurface of virosomes can be readily modified. The use of immune potentiatingreconstituted influenza virosomes (IRIV) ${ }^{9,10}$ broadly applicable with almost all important drugs (anticancer drugs, proteins, peptides, nucleic acids, antibiotics, fungicides). It enables drug delivery into the cytoplasm of target cell, promotes fusion activity in the endolysosomal pathway and protects drugs against degradation.

\section{Dissimilarityas of Liposomes:}

Liposomes have been measuredhopeful vehicles for targeting and liberation of biologically active molecules to living cells in cooperation in vitro and in vivo. However, liposomes have modestimpending to fuse with cells and thus, generally fail to make availablesubstantialrelease of encapsulated molecules to the cell cytoplasm. In disparity, virosomesrestrainserviceable viral envelope glycoproteins with receptor-binding and membrane-fusion properties that facilitate the cellular delivery of encapsulated molecules. ${ }^{11}$

\section{Imperviousretort:}

Virosomessymbolizeanpioneering drug-delivery system for an assortment ofpurely active molecules, but principally nucleic acids or genes, and for numerous indications. Influenza virosomes are virus-like particles, which hang on to the cell fastening and membrane fusion properties of the inhabitant virus, but lack the viral genetic substance. Theseserviceabledistinctiveness of influenza virosomesoutward appearance the foundation for their immunogenicity. Foremost, the rhythmic arrangement of the major viral packet glycoprotein, haemagglutinin, on the virosomalsurfacemediates a co-operative interaction with Ig receptors on B lymphocytes, invigorating strong antibody responses. The envelope proteins (thepeplomers) of enveloped viruses are the components that are important for induction of protective immunity ${ }^{12}$. The opportunity of synthesizing hefty amounts of any preferred protein in bacteria, yeast or animal cells by means of recombinant DNA technology, subunit vaccines are attracting renewed interest ${ }^{13}$. For enveloped viruses the spike glycoproteins are the antigens accountable forstimulating shieldingimmunity. Spike glycoproteins are amphiphilic proteins in which at least one subunit of the oligomeric protein is inserted into the lipid bilayer of the viral envelope and anchors the spike protein to the membrane ${ }^{14}$. To fabricate a subunit vaccine containing only spike proteins, the proteins have to be secluded from the virus and freed from other virus components ${ }^{15}$.

\section{Preparation of virosomes:}

Virosomes made from influenza virus retain the cell entry and membrane fusion capacity of this virus 16,17. Functionally reconstituted influenza virosomes will bind to sialic acid residues on the surface of cells and enter the cell via receptormediated endocytosis ${ }^{18,19}$. Upon endocytosis, the low $\mathrm{pH}$ in the endosomes induces fusion of the virosomal membrane with the endosomal membrane, causing the release of the contents of the virosome into the cytoplasm of the cell. The fusion process is mediated by hemagglutinin, the major envelope glycoprotein of influenza virus ${ }^{20,21}$. These procedures involved in the insertion of influenza HA and NA into immunostimulating complexes (ISCOMS) ${ }^{22,23}$, plain liposomes, liposomes containing immunomodulators such as muramyldipeptide ${ }^{24,25}$, or liposomes containing cationic lipids 26 .

Immunopotentiating reconstituted influenza virosomes (IRIVs) are prepared by the detergent removal of influenza surface glycoproteins, hemagglutinin (HA) and neuraminidase (NA), which are subsequently combined with natural and synthetic phospholipids. The phospholipids consist of $70 \%$ lecithin, a structural component, $20 \%$ cephalin, a structural component that also stimulates B cells independently of $\mathrm{T}$-cell determinants and can bind hepatitis A antigen, and $10 \%$ envelope phospholipids, originating from the selected influenza virus. The resulting IRIVs are spherical, unilamellar vesicles with a mean diameter of approximately $150 \mathrm{~nm}$ and, due to the low viral and avian protein content, are a virtually non-immunogenic delivery system.

\section{Preparation of virosomes for drug delivery:}

To plan virosomes, a viral membrane-fusion protein such as $\mathrm{HA}$ - the generally preferred fusion protein for virosomes - is either purified from the analogous virus or fashioned recombinantly. The triumph of virosomes as a vaccine or delivery vehicle requires that reconstituted membrane proteins keep hold of their immunogenic properties as well as their receptor-binding and membranefusion activities. This involves purposeful reconstitution of influenza virus membranes, which is based on solubilizing viral membranes by nondenaturing detergents. Influenza virus envelopes incorporated with HA can be solubilized with nonionic detergents having a relatively low critical micellar concentration (CMC). Octaethylene glycol mono (n-dodecyl) ether (C12E8) is the most commonly used detergent. Triton X-100 is a frequently used alternative detergent. Other nonionic detergents also can be used. ${ }^{27}$

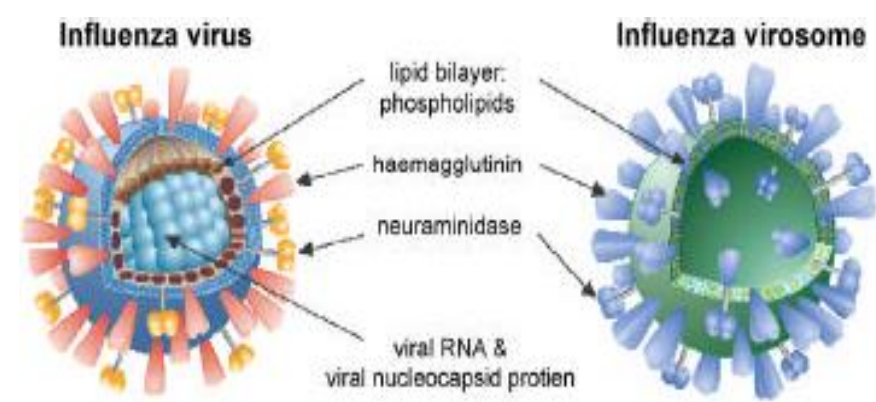

Figure 1: Reconstituted Influenza virosomes 


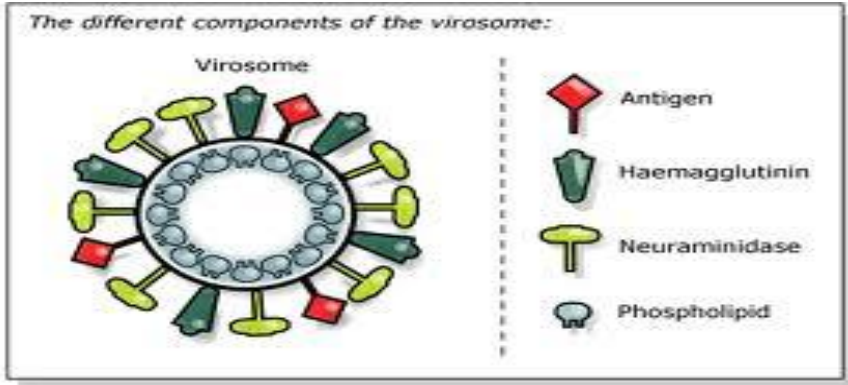

Figure 2: Different components of virosome

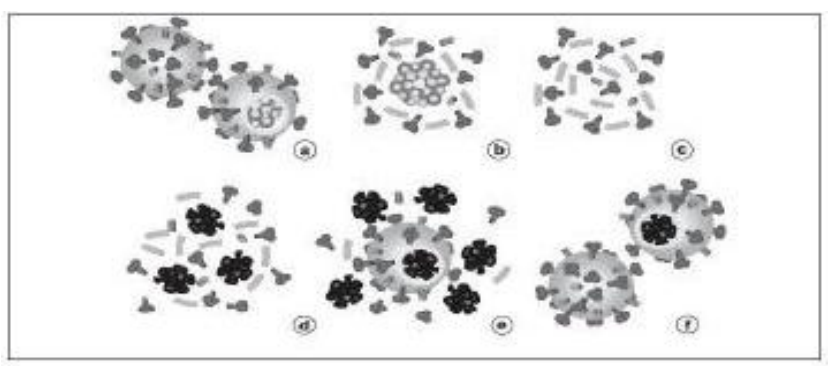

Figure 3: Different stages of preparation of Inflenzavirosomes (a-f)

The perception of conferring viral functions to liposomes will show the way to ainnovativeground in drug delivery originalvirosome vectors will acquire the properties of competent delivery consequential from viral molecules and of lessened toxicity derived from liposomes. In recent times, a new approach, constructing influenza virosomes with a cationic lipid was reported. Influenza virus A was solubilized with detergent and assorted with a cationic lipid, dioleoyldimethylamonium chloride (DODAC), at $30 \%$ to form a cationic virosomes. Plasmid DNA was complexed with these cationic virosomes and was fruitfully transferred to cultured cells by low $\mathrm{pH}$-dependent membrane fusion. ${ }^{28}$

Pellet is resuspended and left overnight to allow for complete solublisation of viral membrane. The suspension is homogenized and ultra centrifuged to pellet down viral nucleocapsids Proteins are added to the supernatant upon detergent extraction using biobeads the virosomes areform Virosomes suspension is purified by ultracentrifugation method. The virosomes can be dialyzed against buffer and sterilized by filtration.

\section{Mode of action:}

Virosomes operate both as a carrier and as an adjuvant, with multiple functions for the duration of initiation of an immune response. The carrier function comprises the affirmative effects of embedding the antigen into a privileged structure, the virosome particle. The adjuvant task relates to immune stimulating properties of the virosomes and their components on the immune system. Most importantly, virosomes accomplish something in stimulating specific immunity without causing nonspecific inflammation.

\section{Carrier}

\section{function:}

The integration of the antigen into the higher structure of the virosome particle stabilizes the antigen, preserves the native status of $\mathrm{B}$ cell epitopes, and protects the antigen from degradation. The antigen displayed on the virosomal surface mimics the original pathogen or target cell and thereby favors the generation of antibodies relevant for protection. Moreover, the presentation of the antigen as a repetitive surface structure enhances its recognition by antibody-producing B cells. Finally, the size and surface structure of the virosome particles make them an attractive target for uptake and processing by immune cells, which is a crucial step in the initiation of an immune response.

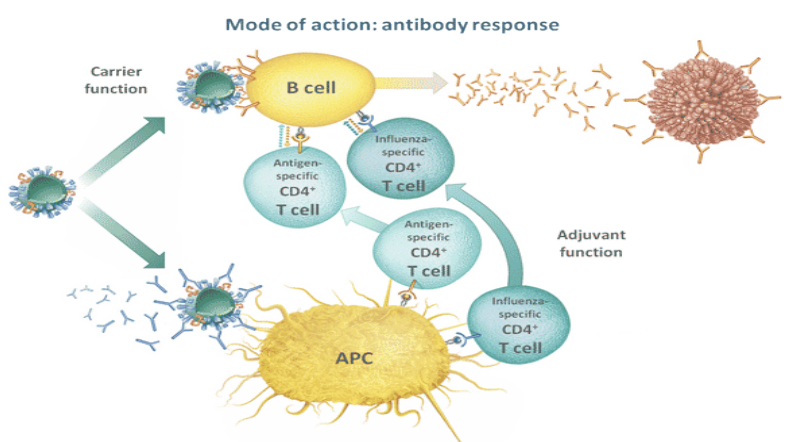

Figure 4: Mechanism of action: antibody response

Evaluation parameters of Virosomes:

\section{Structure and size:}

Negative-stain electron microscopy can generally be used to determine the ultrastructure and size of virosomes. The staining solutions ought to preferably be of neutral $\mathrm{pH}$, to avoid acid-induced conformational changes of $\mathrm{HA}^{29}$

\section{Fusion activity:}

Generally virosomesdemonstrate $\mathrm{pH}$-dependent membrane fusion activity analogous to native influenza virus. Virosomal fusion with biological or artificial intention membranes can be visualized with a fluorescent resonance energy transfer assay (RET). ${ }^{30}$ Instead, fusion can be assessed in vitro with an excimer assay using pyrenelabeled lipids, where the dwindle of surface density of the pyrene-phosphatidylcholine-label on fusion with an unlabeled membrane corresponds to a diminution of excimer fluorescence. ${ }^{29}$ Fusion activity also can be indirectly monitored by determining hemolytic activity, which corresponds intimately to fusion activity and exhibits a $\mathrm{pH}$ dependence identical with that of fusion.

\section{Protein detection:}

Virosome preparation should generally conclusion in a relativelystandardized protein-to-lipid proportion. Sodium dodecyl sulfate-polyacrylamide gel electrophoresis (SDSPAGE) can substantiate the pervasiveness of HA protein in the virosomes. ${ }^{30}$

\section{Specific delivery of nucleic acids/ genes:}

Bioactive drug compounds can be entrapped in the aqueous interior of the virosome or in the lipid membrane of the virosome for facilitated entry of the compounds into the cells. ${ }^{31}$ 
Virosomes are particularly useful for delivering nucleic acids or genes. These compounds are delivered into the host cell cytoplasm on fusion of the virosome with the endosome or plasma membrane. ${ }^{20}$ Nucleic acids or genes encoding a naturally occurring protein can be introduced into host cells and can be expressed, provided that the expression cassette contains the proper cis-acting regulatory elements. ${ }^{32,33}$

Drugs or nucleic acids can be incorporated into the virosome at the time of virosome preparation. The bioactive compound is typically added to the lipid-HA-containing solution following removal of the nucleocapsid. Alternatively, the bioactive compound is initially incorporated into a liposome, which is then fused with a virosome containing two hemagglutinins with different $\mathrm{pH}$ thresholds to form a virosome-liposome hybrid. ${ }^{34}$

\section{Targeted delivery of proteins:}

Proteins also can be delivered to cells via virosome. For example, the gelonin subunit A of diphtheria toxin and ovalbumin have also been effectively delivered by virosome to target cells. ${ }^{34,35}$ Virosomes carrying peptides consequential from the influenza nucleoprotein or intact ovalbumin induced strong cytotoxic $\mathrm{T}$ lymphocyte responses, which suggests that the encapsulated peptides and proteins gained access to the cytoplasm. ${ }^{36,37}$

\section{Carrier for antigen delivery:}

The potential for virosomes as delivery systems for peptide and nucleic acid based vaccines has been investigated for several diseases including malaria, melanoma, hepatitis $\mathrm{C}$ virus and Alzheimer's disease ${ }^{38}$. When virosomes are to be used for the delivery of antigen into the cytosol of cells, fusion activity of the virosomes is of major significance. Studies have shown that virosomes as a delivery system are apposite for the resourceful induction of antibody responses against conformational epitopes by use of cyclic-template bound peptidomimetics. Virosomes as antigen carriers both defend the incorporated peptide and adjuvants and make available additional immunogenicity. By virtue of the fact that reconstituted viral envelopes closely mimic the outer surface of the virus they are derived from, virosomes also symbolize a very useful system for the initiation of antibody responses against the native virus ${ }^{39}$. Furthermore, virosomes may also be used to integrate other unrelated antigens in the virosomal membrane.

\section{Conclusion:}

The challenge of novel vaccine development remains to design vaccine formulations that closely mimic a specific pathogen without being pathogenic themselves. The generation of reconstituted virus envelopes is a promising step in this direction. Virosomes derived from influenza virus can interact with the immune system in variousways eventually leading to activation of Bcells, dendritic cells and T cells as observed during virus infection ${ }^{40}$. Virosomesretain the functional capacity of the original virus to bind to and enter cells andto fuse with limiting of the endosomal cell compartment such that the virosomecontent is delivered to the cytosol. This capacity opens the possibility todeliver antigens to the cytosol of antigen-presenting cells. These antigens can beprocessed and presented by MHC class I molecules to CD8+ $\mathrm{T}$ lymphocytesthus extending theimmuneresponse elicited. In the context of improved influenzavaccines, CTL activity directed against the major internal antigens of thevirus, NP and M1, may be induced in this manner. Finally, virosomes representan excellent platform for inclusion of specific lipophilic or amphiphatic adjuvantsfor further improvement of the quality and quantity of the inducedimmune response ${ }^{47}$. With the new possibilities for mass production of virus antigens, theinterest in subunit vaccines has increased tremendously. There is no doubt thatwe have entered a new era in the development of vaccines. It will now bepossible to engineer viral antigens such that they will evoke an efficient immuneresponse with a maximum of safety. However, careful work is still needed toestablish the optimal way of presenting the relevant antigenic determinants tothe immune system of the host to induce humoral or mucosal immunity. Moreover,virosomes may also improve transfection efficiency by increasing theretention time and reducing the rate of DNA degradation by extracellular nucleases ${ }^{41,42}$.Thus, the virosomes appear to be an effective tool for targeting andgene delivery, and they provide a novel promising approach for the developmentof efficious human vaccines. On the basis of their flexibility and stability,simple production and distinctive immunogenic properties, VLPs offer vastopportunities of application in the fields of vaccine development, gene therapyas well as nanobiotechnology9. Many lessons have been learned from VLPbasedtechnologies, which will certainly find themselves confronted by newchallenges in the near future, such as the demand for innovative biomaterials orpotent vaccines for newly emerging diseases ${ }^{43}$. In this light it appears almostironical that viruses as such may serve a good purpose in the biotechnologicalera exploiting their weapons to beat them at their own game.

\section{Future prospects:}

Ideally one would like to be capable to target drug delivery to preferred tissues. One can adaptvirosomes to targets by incorporating precise molecules (e.g., Fab fragments and ligands) into the virosome'smasterpiece. The achievability of targeted delivery of anticancer drugs by means of virosomal carrier has been confirmed recently by two independent approaches. In one, a MAb cross-linked to the surface of virosomes mediated specific targeting of the virosomal carrier containing an anticancer drug (e.g., doxorubicin) to human cancer cells. MAbs can attachin particular to cancer-related antigens, provided that a means to target systemically administered virosomes to cancerous tissues. on the other hand, ligands that bind surface receptors on the target cells also can be hop to the virosomes to achieve targeted drug delivery. Tumors of mice treated with targeted drug-loaded virosomes failed to grow, and mortality of these animals was significantly reduced. These positive results will definitely open a new field of applications for virosomal technology. ${ }^{29,31}$

Virosomes represent an innovative drug-delivery system for various biologically active molecules, but chiefly nucleic acids or genes, and for numerous indications. The surface of virosomes can be rightfully modified to make easy targeted drug delivery. However, their wide-ranging pharmacokinetic profile, bioavailability, clinical effects, and stability should be premeditated thoroughly to ascertain their long-term reliability as a safe, effective, and reasonable means for drug delivery and targeting. 


\section{REFERENCES:}

1. Storma G, Targeting influenza virosomes to ovarian carcinoma cells, FEBS, 509, 2001, 71-76.

2. Almeida JD, Brand CM, Edwards DC, Heath TD. Formation of virosomes from influenza subunits and liposomes.Lancet.1975; 2:899-901.

3. Zurbriggen R, Immunostimulating reconstituted influenza virosomes, Vaccine, 21, 2003, 921-924.

4. Norrby E, Viral vaccines: The use of currently available products and future developments, Arch. Viral, 73, 1983, 163-165.

5. Vadolas J, Davies JK, Wright PJ, Strugnell RA., Intranasal immunization with liposomes induces strong mucosal immune responses in mice, Eur J. Immunol , 25, 1995, 969-975.

6. Cusi MG, Zurbriggen R, Bianchi S, Valassina M, Valensin PE, Donati $\mathrm{M}$, et al, Intranasal immunization with mumps virus DNA vaccine delivered by influenza virosomes elicits Cusi MG, Gluck R. Potential of DNA vaccines delivered by influenza virosomes. Vaccine, 18, 2000, 1435.

7. Cusi MG, Gluck R. Potential of DNA vaccines delivered by influenza virosomes. Vaccine,18, 2000, 1435.

8. Hu K-F, Ekstrom J, Merza M, Lovgren-Bengtsson K, Morein B., Induction of antibody responses in the common mucosal immune system by respiratory syncytial virus immunostimulating complexes, Med MicrobiolImmunol, 187, 1999, 191-198.

09. Gluck R, Mischler M, Brantschen S, Just M, Althaus B, Cryz SJ., Immunopotentiating reconstituted influenza virosome (IRIV) vaccine delivery system for immunization against hepatitis A, J. Clin Invest, 90, 1992, 2491-2495.

10. Gluck R. Adjuvant activity of immunopotentiating reconstituted influenza virosomes (IRIVs). Vaccine, 17, 1999, 1782-1787.

11. Kaneda Y. Virosomes: evolution of the liposome as a targeted drug delivery system. Adv Drug Deliv Rev. 2000; 43: 197-205.

12. Kammer AR, Amacker M, Rasi S, Westerfeld N, Gremion C, Neuhaus D, Zurbriggen R, A new and versatile virosomal antigen delivery system to induce cellular and humoral immune responses, Vaccine 25 (2007) 7065-7074.

13. Morein B, Simonst K, Subunit vaccines against enveloped viruses: virosomes, micelles and other protein complexes, Vaccine, Vol. 3, June 1985, 83-93,

14. Gluck R. Adjuvant activity of immunopotentiating reconstituted influenza virosomes (IRIVs). Vaccine, 17, 1999, 1782-1787.

15. Kaneda Y, Virosomes: evolution of the liposome as a targeted drug delivery system, Advanced Drug Delivery, 43, 2000, 197205.

16. Bron R, Ortiz A, Dijkstra J, Stegmann T, Wilschut J. Preparation, Properties and applications of reconstituted influenza virus envelopes (virosomes). Methods Enzymol, 220, 1993, 313-331.

17. Stegmann T, Morselt HW, Booy FP, van Breemen JF, Scherphof G, Wilschut J. Functional reconstitution of influenza virus envelopes, EMBO J, 6, 1987, 2651-2659.

18. Matlin KS, Reggio H, Helenius A, Simons K., Infectious entry pathway of influenza virus in a canine kidney cell line. J Cell Biol, 91, 1981, 601-613.

19. Lanzrein M, Schlegel A, Kempf C., Entry and uncoating of enveloped viruses, Biochem J., 302(Part 2), 1994, 313-20.

20. Wilschut J, Bron R. The influenza virus hemagglutinin: membrane fusion activity in intact virions and reconstituted virosomes. In: BentzJ, editor. Viral fusion mechanisms. Boca Raton: CRC Press, 1993, 133-61.

21. Skehel JJ and Wiley DC., Receptor binding and membrane fusion in virus entry: the influenza hemagglutinin, Annu Rev Biochem, 69, 2000, 531-69.

22. B. Morein, D. Barz, U. Koszinowski, V. Schirrmacher, Integration of a virus membrane protein into the lipid bilayer of target cells as a prerequisite for immune cytolysis. Specific cytolysis after virosome target cell fusion, J. Exp. Med. 150, 1979, 1383- 1398.

23. A. Coulter, R. Harris, R. Davis, D. Drane, J. Cox, D. Ryan, P. Sutton, S. Rockman, M. Pearse, Intranasal vaccination with ISCOMATRIX adjuvanted influenza vaccine, Vaccine 21, 2003, 946- 949.
24. K. Nerome, Y. Yoshioka, M. Ishida, K. Okuma, T. Oka, T. Kataoka, A. Inoue, A. Oya, Development of a new type of influenza subunit vaccine made by muramyldipeptideliposome: enhancement of humoral and cellular immune responses, Vaccine, 8, 1990, 503-509.

25. H. Iinuma, K. Nerome, Y. Yoshioka, K. Okinaga, Characteristics of cytotoxic $\mathrm{T}$ lymphocytes directed to influenza virus haemagglutinin elicited by immunization with muramyldipeptideinfluenza liposome vaccine, Scand. J. Immunol, 41, 1995, 1- 10.

26. R. Glqck, R. Mischler, S. Brantschen, M. Just, B. Althaus, S.J. Cryz Jr., Immunopotentiating reconstituted influenza virus virosome vaccine delivery system for immunization against hepatitis A, J. Clin. Invest. 90, 1992, 2491-2495.

27. Stegmann T, Morselt HWM, Booy FP, Van Breemen JFL, Scherphof G, Wilschut J. Functional reconstitution of influenza virus envelopes. EMBO J. 1987;6:2651-9.

28. Waelti E, Wegmann N, Schwaninger R, Wetterwald A, Wingenfeld C, Rothen-Rutishauser B, Gimmi CD: Targeting her$2 /$ neu with antiratNeuvirosomes for cancer therapy. Cancer Res., 62, 2002, 437-444

29. Felnerova D, Viret JF, Glück R, Moser C. Liposomes and virosomes as delivery systems for antigens, nucleic acids and drugs. CurrOpinBiotechnol. 2004;15:518-29.

30. Huckriede A, Bungener L, Stegmann T, Daemen T, Medema J, Palache AM. The virosome concept for influenza vaccines. Vaccine. 2005;23:S26-38.

31. Cusi MG. Applications of influenza virosomes as a delivery system. Human Vaccines.2006; 2:1-7.

32. Daemen T, de Mare A, Bungener L, de Jonge J, Huckriede A, Wilschut J. Virosomes for antigen and DNA delivery. Adv Drug Deliv Rev. 2005; 57:451-63.

33. Sarkar DP, Ramani K, Tyagi SK. Targeted gene delivery by virosomes. Methods Mol Biol. 2002; 199: 163-73.

34. Schoen P, Chonn A, Cullis PR, Wilschut J, Scherrer P. Gene transfer mediated by fusion protein hemagglutinin reconstituted in cationic lipid vesicles. Gene Ther. 1999;6:823-32.

35. Bungener L, Serre K, Bijl L, Leserman L, Wilschut J, Daemen T, Machy P. Virosome-mediated delivery of protein antigens to dendritic cells. Vaccine.2002; 20:2287-95.

36. Arkema A, Huckriede A, Schoen P, Wilschut J, Daemen T. Induction of cytotoxic $\mathrm{T}$ lymphocyte activity by fusion-active peptide-containing virosomes. Vaccine. 2000;18: 1327-33.

37. Bungener L, Huckriede A, de Mare A, de Vries-Idema J, Wilschut J, Daemen T. Virosome-mediated delivery of protein antigens in vivo: efficient induction of class I MHC-restricted cytotoxic T lymphocyte activity. Vaccine. 2005;23:1232-41.

38. YasufumiKaneda, Virosomes: evolution of the liposome as a targeted drug delivery system, Advanced Drug Delivery, 43, 2000, 197-205.

39. A. Huckriede, L. Bungener, W. ter Veer, M. Holtrop, T. Daemen, A.M. Palache, J. Wilschut, Influenza virosomes: combining optimal presentation of hemagglutininimmunopotentiating activity, Vaccine, 21, 2003, 925-931.

40. J. Wilschut, Influenza vaccines: The virosome concept, Immunology Letters, 122, 2009, 118-121.

41. Meyer KB, Thompson M, Levy M, Barron L, Szoka FJ. Intratracheal gene delivery to the mouse air-way: characterization of plasmid DNA expression and pharmacokinetics, Gene Ther, 2, 1995, 450-60.

42. Dzau VJ, Mann MJ, Morishita R, Kaneda Y. Fusigenic viral liposomes for gene therapy in cardiovascular diseases. ProcNatlAcadSci USA, 93, 1996, 11421-11425.

43. R. Gluck, I.C. Metcalfe, New technology platforms in the development of vaccines for the future, Vaccine, 20, 2002, B10B16. 